\title{
Impacto de la formación inicial docente intercultural. Una revisión de la investigación
}

\author{
Impact of intercultural teaching induction training. \\ A review of research \\ Eva Francisca Hinojosa-Pareja / ehinojosa@uco.es \\ Universidad de Córdoba, España \\ Ma Carmen López-López / mclopez@ugr.es \\ Universidad de Granada, España
}

\begin{abstract}
The theoretical and normative advance of multicultural education has stressed the need to adapt preservice teacher education to the plural nature of social and educational reality. This article focuses on the empirical research conducted between 2005 and 2012 and aimed to analyze the impact of preservice teacher education programs committed to cultural diversity on future education professionals. Findings provide information on key factors that influence the success or failure of teacher training proposals, as well as training actions that have the most impact and the methodological limitations most frequent in this research area.
\end{abstract}

Key words: teacher education, preservice teachers, cultural diversity, review of the literature, educational research.

Resumen: El avance teórico y normativo de la educación en y para la diversidad cultural ha subrayado la necesidad de adecuar la formación inicial del profesorado al carácter plural de la realidad social y educativa. Este artículo centra su atención en las investigaciones empíricas desarrolladas entre 2005 y 2012 y destinadas a analizar el impacto que tienen los programas de formación inicial docente comprometidos con la diversidad cultural en los futuros profesores. Los resultados proporcionan información sobre los factores clave que influyen en el éxito o fracaso de las propuestas de formación, así como de las acciones formativas que tienen más impacto y de las limitaciones metodológicas más frecuentes de esta línea de investigación.

Palabras clave: formación del profesorado, futuros docentes, diversidad cultural, revisión de la literatura, investigación educativa. 


\section{Introducción}

Son numerosos los discursos y declaraciones internacionales que apelan a la necesidad de adecuar la formación inicial del profesorado a los cambios sociales e incrementar su sensibilidad y compromiso con la diversidad cultural (Arnesen et al., 2008; Comisión Europea, 2010). Según la Unesco (2009), el mayor avance en el pensamiento educativo internacional es, precisamente, el reconocimiento de las múltiples interpretaciones de la realidad y las diversas formas de enseñar y aprender.

Sin embargo, el gran desafío educativo en estos momentos no se encuentra exclusivamente en el reconocimiento de la diversidad cultural, sino en cómo construir una sociedad inclusiva y una educación para todos que impulse el intercambio cultural y el bien común (CIDE, 2005).

Este creciente interés por la inclusión de la diversidad cultural, impulsada en la declaración de principios, parece tener, no obstante, una limitada repercusión en los programas de formación inicial del profesorado (Sleeter, 2001; Zeichner, 2009), donde su incidencia es escasa y parcial, incluso en aquellas iniciativas en las que existe un compromiso expreso con la diversidad cultural (Morrier et al., 2007). Como han señalado Aguado et al. (2008), la incorporación del componente multicultural a los programas de formación docente es inexistente o suele realizarse mediante la introducción de iniciativas esporádicas y materias, generalmente, optativas que resultan insuficientes.

Por otra parte, existen serias dudas sobre el enfoque teórico adoptado, pues, como destaca Gorski (2006), la formación del profesorado suele articularse desde un punto de vista eurocéntrico, marcado por teorías del déficit que conciben la diversidad cultural como problema. Estas concepciones, con frecuencia, vienen acompañadas de respuestas formativas folclóricas y medidas de corte asimilacionista que poco contribuyen a la igualdad y al enriquecimiento mutuo.

Estudios rigurosos de este hecho han subrayado la escasez de análisis integrales que contemplen la dimensión social y política de la enseñanza y los problemas asociados a la desigualdad del alumnado (Banks et al., 2005; Matus e Infante, 2011). La adopción de este tipo de enfoques comporta la incorporación de perspectivas más reflexivas y complejas que ayuden a los futuros docentes a valorar críticamente la incidencia de las condiciones sociales, económicas y políticas en la construcción de la identidad y el desarrollo personal y grupal. En opinión de Gay (2013), se requiere un tratamiento a largo plazo en el que la diversidad cultural impregne a todos y cada uno de los elementos del currículum y al profesorado en su conjunto. 
A pesar de las dificultades que esto entraña, cada vez son más las iniciativas dirigidas a mejorar la formación inicial del profesorado en y para la diversidad cultural (Sales, 2006; He y Cooper, 2009; Waddell, 2011), y más las investigaciones y producción científica que centra su interés en esta temática (Grant y Secada, 1990; Cochran-Smith et al., 2004; Grant y Gibson, 2011).

Así surge una línea de investigación, consolidada en el marco de la formación docente (OCDE 2010), dirigida a desvelar el impacto de los programas de formación inicial del profesorado en y para la diversidad cultural en los futuros docentes. Este trabajo recoge, precisamente, los progresos experimentados en este campo mediante la revisión de trabajos de investigación empírica desarrollados durante el periodo 2005-2012.

\section{Procedimiento}

Es una investigación bibliográfica que, como ha señalado Bisquerra (1989), va orientada a la búsqueda, recopilación, organización, valoración y crítica de información bibliográfica sobre temas específicos. Para este autor, una de las mayores virtualidades de este tipo de investigación es que "permite la difusión de una visión panorámica del problema a todos los interesados en el tema" (Bisquerra, 1989: 68).

Para revisar la producción científica se ha empleado el análisis documental, entendido como conjunto de acciones destinadas a analizar y representar el contenido y la forma de un documento (Clauso, 1993). El procedimiento de análisis ha contemplado el doble nivel descrito por Pinto (1989): análisis de elementos formales y análisis interno o de contenido.

El primer nivel ha consistido en la localización y descripción bibliográfica de la documentación (durante el mes de febrero de 2013). Se trata de un nivel más cercano a la catalogación, donde se han localizado los documentos y clasificado en función de sus aspectos físicos (fuente, autoría, año y lugar de publicación, referencia bibliográfica). Para acceder a la producción científica se han consultado diferentes bases de datos y manuales especializados.

En el contexto internacional se han considerado las bases de datos ERIC y SCOPUS, así como los manuales compilatorios de Ball y Tyson (2011), OCDE (2010) y Townsend y Bates (2007). Los descriptores utilizados para la búsqueda han sido: teacher education, preservice, diversity y multicultural/ intercultural. La producción científica de ámbito nacional ha sido revisada a partir de las bases de datos CSIC-ISOC, DIALNET y TESEO mediante los siguientes descriptores: formación, profesorado/docente, diversidad cultural y 
multicultural/intercultural. Con estos parámetros se obtienen 320 documentos, 199 correspondientes al contexto internacional y 121 al nacional.

El segundo nivel, de análisis interno, ha estado compuesto de dos fases. La primera de ellas, desarrollada entre marzo y abril de 2013, ha consistido en la lectura del título, resumen y descriptores de los documentos, con la intención de identificar y seleccionar los estudios que atienden a los criterios siguientes: a) investigaciones empíricas, $b$ ) trabajos cuyo objeto es estudiar el impacto que tienen las iniciativas de formación inicial del profesorado sobre diversidad cultural en los futuros docentes. Estos criterios han permitido reducir a 55 el número de documentos que han servido de base a nuestra revisión. Las revistas en las que se encuentran los artículos analizados pueden consultarse en el Cuadro $1 .^{1}$

La segunda fase ha sido la más intensa (realizada entre mayo y junio de 2013). Se trata de un momento de lectura en profundidad y análisis del contenido de los estudios en torno a los siguientes elementos: problema de investigación, diseño metodológico, técnicas e instrumentos de recogida de información, muestra/participantes y resultados.

Una vez analizados los documentos, se han organizado en función de los espacios donde se lleva a cabo la formación inicial. Como apuntan Biddle, Good y Goodson (2000), los contextos de enseñanza son determinantes en el impacto que tiene la formación inicial en los futuros docentes y los aprendizajes que desarrollan. Asimismo, también es un criterio de clasificación empleado en revisiones previas (Sleeter, 2001; Hollins y Torres, 2005), que diferencian entre propuestas formativas desarrolladas sólo en centros universitarios e iniciativas que incluyen experiencias de campo o de inmersión en contextos multiculturales. Con todo, se han establecido dos tipos de investigaciones:

- Estudios centrados en el impacto de la formación desarrollada en centros de formación inicial del profesorado.

- Investigaciones sobre el impacto de propuestas formativas que incluyen experiencias de inmersión en la realidad multicultural.

\section{Resultados}

A continuación se presentan los resultados más destacados de la revisión efectuada en función de las tipologías de investigaciones establecidas.

Estudios sobre el impacto de propuestas formativas desarrolladas en centros de formación inicial del profesorado.

1 Este cuadro se encuentra en el Anexo, al final del presente texto (Nota del editor). 
Esta sección recoge 28 trabajos de investigación destinados a conocer el impacto de la formación en y para la diversidad cultural desarrollada en centros de formación inicial del profesorado sobre los futuros docentes.

Tomando como referencia el contexto internacional, destacamos, con resultados positivos, los trabajos de Guidry y Sowa (2005) y Wasonga (2005). Los primeros centran su atención en el análisis de contenido de los diálogos on line entre estudiantes y profesorado de distintos orígenes culturales. El estudio desvela que la experiencia ha contribuido a ver el mundo desde diferentes ópticas, a entender la diversidad cultural como una cualidad inherente al ser humano, y a comprender que la visión de la realidad es limitada y está ligada a la propia experiencia.

Wasonga (2005), por su parte, estudia, mediante encuestas, la influencia de una experiencia formativa sobre el conocimiento y las actitudes de los futuros docentes y la relación entre ambas variables. En la investigación se concluye que la formación para la diversidad cultural influye notablemente en el conocimiento y en las actitudes, pero que ambas no están vinculadas entre sí, por lo que el conocimiento no se puede usar como predictor del cambio actitudinal.

Paredes (2009), en un estudio cuantitativo y cualitativo, encuentra resultados igualmente positivos y subraya que esta formación influye especialmente en el conocimiento del contenido, de la lengua y pedagógico. Cho y DeCastro (2006) se interesan por conocer, mediante cuestionario, el cambio actitudinal de los futuros docentes. Los resultados, aunque positivos, son tomados con cautela porque, junto a actitudes favorables, hay estudiantes que niegan la presencia de racismo y no consideran beneficiosa la atención a la diversidad cultural.

Tsigilis et al. (2006) estudian la variación de actitudes y su relación con el comportamiento apoyándose en las respuestas de 148 estudiantes a un cuestionario. Los resultados evidencian una mejora de las actitudes hacia diversidad cultural y que éstas se pueden predecir, en parte, por el comportamiento. Szecsi et al. (2010), interesados también en desvelar el impacto de la formación en las actitudes de los estudiantes, encuentran que ésta ha tenido una influencia positiva en cuanto a una mayor conciencia sobre la cultura y la necesidad de ampliar la formación docente para la diversidad cultural.

Otras investigaciones se preocupan por el impacto de la formación sobre las concepciones, creencias y opiniones de los futuros docentes. A este grupo pertenecen los trabajos de Fox y Diaz-Greenberg (2006), Moore (2009), Kinloch (2011), Laughter (2011) y Liggett (2011) quienes, mediante aproximaciones cualitativas, análisis de documentos, entrevistas y observaciones 
de clase, afirman que la formación docente para la diversidad cultural tiene un impacto importante en la modificación de las concepciones previas y las creencias, genera una comprensión más profunda de la cultura, significados más amplios de la enseñanza en contextos multiculturales y una concienciación crítica incipiente. Estos autores consideran que la reflexión, el intercambio con compañeros y el trabajo colaborativo son herramientas de éxito claves en los programas formativos.

En los estudios de caso desarrollados por Milner (2006), Christensen (2007), McKnight et al. (2011) y Patchen (2012) se analizan los aprendizajes y conocimientos adquiridos durante la formación. En el caso de Milner (2006), se concluye que las actividades dirigidas a impulsar la concienciación, reflexión crítica y la vinculación entre teoría y práctica son las que tienen mayor impacto. Christensen (2007), por su parte, afirma que los futuros docentes han mejorado la comprensión cognitiva de conceptos de educación intercultural y esto incrementa su conciencia y competencia intercultural.

En este estudio se destaca la reflexión de la perspectiva etnográfica adoptada en la formación docente como factor relevante en los progresos experimentados. McKnight et al. (2011) señalan que los participantes de su investigación también han incrementado y mejorado su conocimiento y han adquirido una mayor concienciación sobre la diversidad cultural. Patchen (2012), con el objetivo de mejorar el aprendizaje y evitar la disonancia cognitiva de los futuros docentes, analiza el impacto que tiene el uso del cine como herramienta de reflexión y conocimiento del otro en una asignatura de educación intercultural.

Los resultados confirman que la estrategia utilizada ayuda a comprender mejor la diversidad cultural, sobre todo a estudiantes que han interaccionado poco con personas de distinta procedencia cultural. En este trabajo también se subraya la tendencia de los estudiantes a realizar comparaciones binarias y simplistas cuando describen aspectos relacionados con la diversidad y la variación de respuestas en función de la etnia.

Con propósitos similares figuran los estudios de Lenski et al. (2005), Cain (2010) y Gunn (2011). El primero de ellos analiza el impacto que tiene un proyecto de investigación-acción etnográfico en los futuros profesores. A través de entrevistas y documentos elaborados por 22 estudiantes, los autores descubren que los futuros docentes interactúan desde perspectivas distintas a la propia y examinan más críticamente las situaciones que observan y sus propias creencias. 
Un estudio de seguimiento posterior con seis de estos participantes confirma que los cambios se mantienen durante su primer año de ejercicio profesional. Cain (2010) utiliza la narrativa de ficción on line como estrategia para potenciar la reflexión sobre la diversidad cultural. Combinando una metodología cualitativa y cuantitativa, recoge evidencias de 10 estudiantes durante un semestre.

Sus hallazgos le permiten afirmar que la experiencia formativa ha facilitado la reflexión, concienciación y entendimiento de la multiculturalidad. Gunn (2011), por su parte, examina la pertinencia de los estudios de caso como estrategia de formación para la diversidad de los futuros docentes. Los resultados revelan que la estrategia influye positivamente en las percepciones de la mayoría de los estudiantes, incrementa su motivación y mejora la profundidad de la discusión y la transferencia a la práctica.

Con resultados menos concluyentes encontramos los trabajos de Espiritu (2006), Hill-Jackson et al. (2007) y Fernsten (2009). Espiritu (2006), basándose en las observaciones a 23 estudiantes y en los trabajos realizados en clase, reconoce que la formación para la diversidad cultural tiene impactos diferentes en función de la posición de privilegio social, cultural y económico de los participantes.

Los estudiantes de grupos privilegiados minimizan el poder social de su posición y su contribución en el mantenimiento de las desigualdades socioculturales y actitudes racistas; mientras que los estudiantes en desventaja asumen una postura más crítica hacia la sociedad y la atención proporcionada a la diversidad cultural. Hill-Jackson et al. (2007), en un estudio con 200 alumnos, revelan que el impacto de la formación inicial varía en función del perfil de cada persona.

Los perfiles hallados son dos: defensores y resistentes. Cada perfil presenta distinta complejidad cognitiva, visión del mundo, sensibilidad intercultural, ética y percepción de la autoeficacia, que va a condicionar el impacto de las experiencias formativas y las posiciones que finalmente se adoptan respecto a la diversidad cultural. Fernsten (2009), a partir de la administración de un cuestionario a 48 estudiantes, establece que la incidencia de la preparación docente recibida varía dependiendo del contacto con culturas distintas a la propia y el conocimiento previo que se tenga sobre diversidad cultural.

Otros trabajos muestran resultados aún menos alentadores, pues concluyen que la formación inicial del profesorado no sólo no produce impacto en los estudiantes, sino que, a veces, refuerza estereotipos y/o conocimientos poco sensibles a la diversidad cultural. Locke (2005), por ejemplo, entrevista y observa a 13 estudiantes y concluye que la formación diseñada para cuestionar 
marcos de referencia previos no ha tenido impacto alguno en las respuestas de los futuros docentes, logros que sí han conseguido el entorno social o los medios de comunicación.

De Courcy (2007) realiza una investigación-acción con 37 estudiantes durante un curso de educación multicultural. Los resultados reflejan que se han reforzado concepciones previas desfavorables y, aunque se evidencia que los estudiantes son susceptibles a nuevas ideas, tienden a construirlas en términos de déficit.

También Henkin y Steinmetz (2008) y Szabo y Anderson (2009) alcanzan resultados parecidos. Henkin y Steinmetz (2008), después de aplicar un cuestionario abierto a 56 estudiantes, señalan un nivel bajo de comprensión y concienciación. Szabo y Anderson (2009) constatan que las acciones formativas emprendidas no han tenido la repercusión esperada, pues reflejan un escaso impacto en las concepciones, actitudes y conocimientos previos de los docentes en formación.

Conclusiones similares se encuentran en los trabajos de Berta-Avila y William-White (2010) y Reiter y Davis (2011). El primero de ellos evidencia, mediante narrativas, que los futuros docentes muestran concepciones de la diversidad políticamente correctas, un escaso conocimiento sobre la diversidad cultural y necesitan fuertes soportes para desarrollar procesos de crítica y reflexión. En el estudio de Reiter y Davis (2011), centrado en las respuestas emitidas por 153 estudiantes a un cuestionario, se confirma que la formación recibida tampoco ha tenido incidencia en las actitudes y creencias previas de los aspirantes a docentes.

En el contexto nacional destacamos el estudio etnográfico de Herrada (2010), en el que participan 40 estudiantes. Su propósito es analizar la evolución del término raza en el transcurso de una asignatura relacionada con la diversidad cultural. Tras el análisis de datos obtenidos a través de cuestionario, observaciones y ensayos, la autora constata que los estudiantes problematizan el concepto, se replantean sus creencias y actitudes, y, en algunos casos, extrapolan lo aprendido al contexto del aula.

\section{Investigaciones sobre el impacto de propuestas formativas que incluyen experiencias de inmersión en la realidad}

El número de estudios ubicados en esta tipología asciende a 27. Estos trabajos incluyen experiencias de distintos tipos: propuestas formativas que tienen lugar en centros escolares (prácticum), inmersiones en comunidad local (aprendizaje-servicio) y experiencias en comunidades distintas a la autóctona 
o cross-cultural (inmersión en familias, intercambios, etcétera). Entre ellas, las prácticas en centros escolares son las más numerosas (18 de las 27).

En el contexto internacional destacamos el trabajo de Almarza (2005), quien analiza el impacto del prácticum en 240 futuros docentes durante dos años. Se trata de un estudio cualitativo basado en el modelo realista de Korthagen que recoge el proceso vivido por los estudiantes en la formación y que va desde la frustración inicial hasta la deconstrucción de preconceptos y reconstrucción sustentada en la experiencia multicultural vivida.

En esta investigación se subraya la relevancia del prácticum y la reflexión sobre la práctica en la formación docente en y para la diversidad cultural. El estudio de Czop et al. (2010), centrado en el cambio de actitudes y creencias tras el prácticum, también se llega a conclusiones similares. Los trabajos de Zygmunt-Fillwalk (2005) y Zygmunt-Fillwalk y Leitze (2006) estudian, mediante cuestionario, entrevistas y grupos focales, el cambio de actitudes de dos promociones de estudiantes de un programa formativo que incluye inmersión en un colegio culturalmente heterogéneo.

Los hallazgos reflejan una mejora del conocimiento, las actitudes y las creencias previas que se mantiene durante varios años. Los autores afirman que la inmersión cultural, las relaciones establecidas, la reflexión y el apoyo profesional ofrecido han sido decisivos para el éxito del cambio. Gayle-Evans y Michael (2006) aplican un cuestionario a 32 estudiantes antes y después del prácticum y obtienen resultados positivos en cuanto al nivel de concienciación. Hsu (2009), que analiza el impacto del prácticum apoyándose en las entradas a un blog y la aplicación de una escala antes y después de su realización, también señala una mejora de la reflexión sobre la práctica y las actitudes hacia la diversidad cultural.

Con resultados igualmente positivos figuran los trabajos de Cicchelli y Cho (2007), He y Cooper (2009), Bleicher (2011) y Bodur (2012). Cicchelli y Cho (2007) describen la influencia del prácticum en 61 futuros docentes y concluyen que el cambio de actitudes y creencias es más evidente y significativo en estudiantes blancos. Este hallazgo lleva a los autores a considerar que los aspectos sociales o culturales pueden influir en el impacto que finalmente tienen los programas formativos.

He y Cooper (2009) toman como referencia el modelo ABC de Schmitd y analizan el impacto de una acción formativa que incluye experiencias de campo en centros escolares. Utilizando un diseño cuasiexperimental y una escala Likert, estudian las diferencias entre el grupo experimental y el grupocontrol. Los resultados muestran que el conocimiento y la comprensión multicultural mejora en el grupo que participa en la iniciativa. 
Bleicher (2011), por su parte, estudia las percepciones de 95 docentes en formación a través del análisis de reflexiones, entrevistas y un cuestionario aplicado antes y después de realizar el prácticum. Los hallazgos revelan un cambio significativo en las percepciones y una mejora de la confianza en las propias habilidades.

Bodur (2012) analiza la evolución en las creencias y actitudes de dos grupos de estudiantes mediante un cuestionario y entrevistas. Uno de estos grupos cursa un programa de formación para la diversidad cultural que incluye experiencias de campo en centros educativos, mientras que el otro es el grupo-control. Los resultados muestran que el grupo experimental finaliza su periodo formativo con creencias y actitudes más positivas y una comprensión más profunda de la realidad multicultural.

Otros estudios focalizan su atención en el impacto de prácticas mentorizadas. En el trabajo de Klug et al. (2006) la mentoría es ejercida por un estudiante que tutoriza a otro de cultura distinta a la propia. En el estudio de Zozakiewicz (2010), en cambio, el mentor es un profesor experimentado con competencia intercultural que supervisa al futuro docente durante el prácticum. En ambos casos, aunque con metodologías distintas, se constata una mejora en la comprensión del fenómeno multicultural y en las prácticas con alumnado culturalmente heterogéneo.

Junto a este grupo de estudios figuran otros con resultados menos prometedores. A este grupo pertenece, entre otros, el trabajo de Del Prado et al. (2007). En él se analizan los diarios de campo y entrevistas de 38 futuros docentes. Sus hallazgos indican que los estudiantes se muestran reacios a abordar cuestiones vinculadas con la diversidad cultural, no tienen demasiado conocimiento de otros grupos culturales y creen que lo más correcto e igualitario es un tratamiento lo más homogéneo posible.

Kyles y Olafson (2008) estudian las creencias y actitudes antes y después de la formación práctica mediante la aplicación de varias escalas y el análisis de autobiografías. Las escalas no reflejan cambios, mientras que el análisis cualitativo de las autobiografías evidencia algunas mejoras que los autores vinculan al tipo de experiencias vividas por los estudiantes. Aquellos que tienen experiencias de carácter multicultural desarrollan creencias más favorables.

Settllage et al. (2009) investigan, a través de un diseño longitudinal, los cambios en las creencias de 48 futuros docentes tras cursar el prácticum. Sus resultados confirman que no se aprecian modificaciones. De igual modo, Lockhart (2010) y Zippay (2010) describen las concepciones y creencias que tienen los futuros docentes al comenzar y finalizar la formación práctica. Ambos estudios concluyen que el proceso formativo no ha tenido incidencia. 
Las investigaciones centradas en analizar el impacto de prácticas de inmersión en la comunidad local son, como se ha señalado, menos numerosas. Bell et al. (2007) examinan las concepciones que se desarrollan en una experiencia de servicio a la comunidad mediante un cuestionario, el análisis de ensayos y autobiografías.

Los autores señalan que la experiencia ha permitido desarrollar concepciones más complejas de la diversidad cultural. Bollin (2007), en un estudio cualitativo apoyado en el análisis de los diarios de prácticas de aprendizajeservicio a la comunidad, subraya que este tipo de experiencias contribuyen a desarrollar actitudes y valores de respeto, responsabilidad y confianza, desmantelan estereotipos y mejoran la comprensión del desafío que supone enseñar en aulas heterogéneas.

Davis y Moely (2007), mediante la aplicación de una escala a 217 estudiantes, y Waddell (2011), a través de un estudio de caso con 33 futuros profesores, llegan a las mismas conclusiones. Ambos ratifican que estas prácticas de inmersión desarrollan actitudes favorables a la diversidad cultural, mejoran el conocimiento de uno mismo e incrementan el compromiso con la comunidad. Chang et al. (2011) estudian la influencia de variables contextuales y personales en los estudiantes que cursan prácticas de aprendizaje-servicio.

Para ello, seleccionan una muestra de 212 candidatos a los que administran distintos instrumentos de corte cualitativo y cuantitativo. Los resultados indican que la discusión sobre la práctica es la variable que más influye en los aprendizajes. La influencia de otras variables como el sexo, edad u origen sociocultural no muestran resultados unívocos, fluctuando en función del tipo de resultado de aprendizaje contemplado. En este tipo de estudios se destaca la relación estrecha que se establece con personas de otras culturas, el clima de relaciones y el compromiso del profesorado como principales logros.

Para finalizar, presentamos los estudios que analizan el impacto de las experiencias formativas de inmersión en familias de culturas distintas a la propia o en iniciativas de intercambio estudiantiles. Los estudios revisados corresponden a programas formativos estadounidenses en los que las familias de acogida son de origen italiano, inglés, hondureño y dominicano. Todos ellos se proponen analizar la incidencia de la experiencia formativa en la percepción, creencias, actitudes y conocimientos de los futuros docentes.

Ference y College (2006) señalan que estas experiencias de inmersión contribuyen al crecimiento personal, mejoran el conocimiento y comprensión de los estudiantes, y ayudan a desarrollar actitudes favorables hacia la diversidad cultural. En la investigación de Nero (2009) se ratifican estos hallazgos y se subraya el incremento en la complejidad conceptual y la reflexión 
crítica. Marx y Moss (2011), así como Phillion et al. (2009), confirman estos resultados aportando que este tipo de experiencias también ayudan a trasladar el aprendizaje adquirido a la práctica.

En estos trabajos, sin embargo, se alerta de la necesidad de una planificación cuidada y minuciosa de la experiencia formativa, así como de la relevancia que adquiere el compromiso explícito de la familia de acogida.

A nivel nacional destacamos el estudio de Durán (2011), cuyo propósito es analizar las respuestas ofrecidas por futuros docentes después de realizar el prácticum en un programa de intercambio entre España y Reino Unido. La información recabada a través de la aplicación de un cuestionario a 45 estudiantes ha evidenciado que el impacto de la experiencia depende, en gran medida, del perfil de los participantes. En el caso de los docentes españoles, la mejora en el conocimiento, la conexión con experiencias previas y la confianza en sí mismos ha sido más significativa. El autor lo atribuye al hecho de que era la primera vez que el colectivo de docentes españoles participaba en una experiencia cross-cultural, mientras que los ingleses ya lo habían hecho con anterioridad.

\section{Discusión y conclusiones}

Después de la revisión efectuada, podemos afirmar que el esfuerzo desplegado en el contexto nacional para la consolidación de esta línea de investigación es sustancialmente inferior al compromiso deparado en el marco internacional, como se desprende del número reducido de estudios realizados en nuestro entorno en los últimos años. Este hecho confirma que el campo de la formación inicial del profesorado en y para la diversidad cultural en España continúa siendo un campo de indagación incipiente, residual y con escasa visibilidad.

Esto puede estar vinculado a varios factores: la escasez de financiación en investigación educativa, acusada por la crisis económica que azota al país; la desconsideración de la formación en y para la diversidad cultural como área de investigación preferente, y, finalmente, la dificultad de difusión que, en ocasiones, encuentra este tipo de investigaciones debido a su carácter eminentemente local; circunscrito a muestras poco representativas o materias concretas; a la debilidad metodológica en que, a veces, se sustentan; y al escaso uso de repositorios y otros medios, fuera de los circuitos habituales de publicación, que en otros países, como EUA, son comunes.

Se requiere, sin duda, un mayor compromiso y apoyo institucional para incrementar la oferta formativa dirigida al profesorado en este aspecto (Trent 
et al., 2008; Aguado et al., 2008; Ibáñez, 2012; Gay, 2013) y la investigación destinada a analizar su impacto en los docentes en formación y en su futura práctica profesional (Sleeter, 2001; Rodríguez Izquierdo, 2009; Grant y Gibson, 2011). El hecho de que las investigaciones analizadas hayan centrado su interés en los docentes en formación implica reconocer su papel clave en la mejora del proceso formativo (Feiman-Nemser, 2001; Hammerness et al., 2005).

Desde el punto de vista metodológico, se constata un predominio de estudios que combinan los enfoques cuantitativo y cualitativo, y utilizan, como principales técnicas de recogida de información, las entrevistas, grupos focales, análisis documental, cuestionarios y observaciones de aula. Sin embargo, son escasas las investigaciones que optan por un diseño longitudinal, resultando de esta forma más complejo acceder a los impactos reales que tienen las iniciativas de formación inicial del profesorado en la práctica docente futura o en otros aspectos que pudieran evidenciarse a largo plazo.

Esta limitación se une a otras deficiencias detectadas en los estudios analizados y que han sido igualmente denunciadas, entre otros, por Hollins y Torres (2005) o Grant y Gibson (2011). Nos referimos, por ejemplo, al hecho de que gran parte de estos trabajos se apoyan en muestras pequeñas, poco representativas, que derivan en resultados difícilmente generalizables; a la poca atención deparada a la fundamentación del marco metodológico o las técnicas empleadas y, especialmente, a la escasa información ofrecida en relación con la fiabilidad y validez de los instrumentos con los que se recaba la información.

Respecto a los resultados de este ámbito de investigación, cabe señalar que no son concluyentes, pues, junto a investigaciones que exhiben experiencias de formación con impactos positivos (Wasonga, 2005; Szecsi et al., 2010; Kinloch, 2011), se presentan estudios cuyos hallazgos son menos prometedores (Reiter y Davis, 2011; Fernsten, 2009).

Entre los factores que se subrayan como condicionantes del éxito o fracaso de las iniciativas de formación inicial del profesorado en y para la diversidad cultural figuran: las experiencias, conocimientos y creencias previas y su adecuación a las distintas oportunidades de aprendizaje; las metodologías empleadas en la formación inicial docente; los programas de formación; la posición social, cultural y económica que ocupan los aspirantes a profesores en el contexto social; las relaciones de poder entre clases sociales que configuran las experiencias previas de los estudiantes; el entorno y las relaciones sociales; los medios de comunicación y el contacto con otras culturas. 
Aunque, como hemos afirmado, los resultados de las investigaciones revisadas en este artículo no son concluyentes respecto al impacto de la formación inicial del profesorado en los docentes, sí podemos afirmar que las propuestas formativas que se preocupan por vincular teoría y práctica e incorporar la reflexión crítica, el diálogo, el intercambio con compañeros, los estudios de caso y el trabajo colaborativo suelen tener un impacto más positivo en las actitudes, comportamientos, creencias y perspectivas de los futuros docentes hacia la diversidad cultural.

Este hallazgo contraviene las observaciones expuestas por Pajares (1992) y Richardson y Placier (2001), entre otros, y que cuestionan la incidencia de la formación docente en la modificación de las creencias. Sin embargo, entre todas las propuestas de formación analizadas, las experiencias bien planificadas de inmersión en la realidad multicultural, a través del prácticum o de vivencias asociadas a comunidades culturalmente diversas (aprendizaje-servicio, intercambios,...), son las que parecen tener un impacto más favorable.

Estas experiencias ayudan a tomar conciencia del papel que juegan los estereotipos y superarlos; modifican las creencias; desarrollan actitudes y valores de respeto, responsabilidad y confianza mutua; mejoran el conocimiento de sí mismos, así como la comprensión de la realidad multicultural y el desafío que supone enseñar en aulas culturalmente heterogéneas, además de incrementar el compromiso con la mejora de la práctica y el traslado del aprendizaje adquirido a la práctica del aula (Bollin, 2007; Waddell, 2011; Nero, 2009).

\section{Bibliografía}

Aguado, María Teresa et al. (2008), "El enfoque intercultural en la formación del profesorado. Dilemas y propuestas", en Revista Complutense de Educación, año 19, núm. 2, España: Servicio de Publicaciones de la Universidad Complutense de Madrid.

Almarza, Dario (2005), "Connecting multicultural education theories with practice: a case study of an intervention course using the Realistic Approach in Teacher Education", en Bilingual Research Journal, año 29, núm. 3, Estados Unidos: Routledge.

Arnesen, Anne-Lise et al. (2008), Policies and practices for teaching sociocultural diversity. A Survey report, Strasbourg Cedex: Council of Europe Publishing.

Ball, Arnetha y Cynthia Tyson (2011), Studying diversity in teacher education, Lanham: AERA. Banks, James et al. (2005), “Teaching diverse learners", en Darling-Hammond, Linda y John Bransford [comps.], Preparing teachers for a changing world: what teachers should learn and be able to do, San Francisco: Jossey-Bass.

Bell, Courtney et al. (2007), "We know it's service, but what are they learning? Preservice teachers' understandings of diversity", en Equity and Excellence in Education, año 40, núm. 2, Estados Unidos: Routledge. 
Berta-Avila, Margarita Inés y Lisa William-White (2010), "A conscious agenda for cultivating future teachers' equity and social justice paradigms", en Teacher Education and Practice, año 23, núm. 4, Estados Unidos: Rowman \& Littlefield.

Biddle, Bruce, Thomas Good e Ivor Goodson [comps.] (2000), La enseñanza y los profesores. La enseñanza y sus contextos (II), Barcelona: Paidós Ibérica.

Bisquerra, Rafael (1989), Métodos de investigación educativa: guíapráctica, Barcelona: CEAC. Bleicher, Elizabeth (2011), "Parsing the language of racism and relief: Effects of a shortterm urban field placement on teacher candidates' perceptions of culturally diverse classrooms", en Teaching and Teacher Education, año 27, Estados Unidos: Elsevier.

Bodur, Yasar (2012), "Impact of course and fieldwork on multicultural beliefs and attitudes", en The Educational Forum, año 76, núm. 1, Estados Unidos: Kappa Delta Pi/Routledge.

Bollin, Gail (2007), "Preparing teachers for Hispanic immigrant children: a service learning approach", en Journal of Latinos and Education, año 6, núm. 2, Estados Unidos: Routledge.

Cain, Mary (2010), "Culturally responsive teaching awareness through online fiction", en Multicultural Education \&Technology Journal, año 4, núm. 2, Reino Unido: Emerald.

Chang, Shih-pei et al. (2011), "The multidimensionality of multicultural service learning: The variable effects of social identity, context and pedagogy on pre-service teachers' learning", en Teaching and Teacher Education, año 27, Estados Unidos: Elsevier.

Cho, Grace y Debra DeCastro (2006), "Is ignorance bliss? Pre-service teachers' attitudes toward multicultural education”, en The High School Journal, año 89, núm. 2, Estados Unidos: The University of North Carolina Press.

Christensen, Laurene (2007), "A case study of intercultural development for pre-service language teachers", en Higbee, Jeanne, Dana Lundell e Irene Duranczyk [comps.], Diversity and the Postsecondary Experience, Minneapolis: Center for Research on Developmental Education and Urban Literacy, University of Minnesota.

Cicchelli, Terry y Su-Je Cho (2007), "Teacher multicultural attitudes: intern/teaching fellows in New York City”, en Education and Urban Society, año 39, núm. 3, Estados Unidos: Sage.

CIDE (2005), La atención al alumnado inmigrante en el sistema educativo en España, Madrid: Centro de Investigación y Documentación y Evaluación Educativa.

Clauso, Adelina (1993), "Análisis documental: el análisis formal”, en Revista General de Información y Documentación, año 3, núm. 1, España: Universidad Complutense de Madrid.

Cochran-Smith, Marilyn et al. (2004), "Multicultural teacher education”, en Banks, James y Cherry A. McGee Banks [comps.], Handbook of research in multicultural education, San Francisco: Jossey-Bass.

Comisión Europea (2010), How can Teacher Education and Training policies prepare teachers to teach effectively in culturally diverse settings?, Oslo: Comisión Europea.

Czop, Lori et al. (2010), "Multicultural teacher education: examining the perceptions, practices and coherence in one teacher preparation program", en Teacher Education Quarterly, año 37, núm. 2, Estados Unidos: California Council on Teacher Education.

Davis, Tery y Barbara Moely (2007), "Preparing pre-service teachers and meeting the diversity challenge through structured service-learning and field experiences in urban schools", en Townsend, Tony y Richard Bates [comps.], Handbook of Teacher Education, Países Bajos: Springer. 
Convergencia Revista de Ciencias Sociales, núm. 71, 2016, Universidad Autónoma del Estado de México

De Courcy, Michele (2007), “Disrupting preconceptions: challenges to pre-service teachers' beliefs about ESL children”, en Journal of Multilingual and Multicultural Development, año 28, núm. 3, Estados Unidos: Routledge.

Del Prado, Pixita et al. (2007), "Preservice educators' perceptions of teaching in an Urban Middle School setting: A lesson from the Amistad", en Multicultural Education, año 15, núm. 1, Estados Unidos: Caddo Gap Press.

Durán, Ramiro (2011), "Spanish and British teacher trainees on exchange in primary schools abroad: an intercultural experience in educational settings", en Porta Linguarum, año 15, España: Universidad de Granada.

Espiritu, Patricia (2006), "Questioning the Aloha in a multicultural teacher education course”, en Asia-Pacific Journal of Education, año 26, núm. 1, Singapore: Routledge.

Feiman-Nemser, Sharon (2001), "From preparation to practice: designing a continuum to strengthen and sustain teaching", en Teachers College Record, año 6, Estados Unidos: Teacher College, Columbia University.

Ference, Ruth y Berry College (2006), "Building and sustaining short-term cross-cultural immersion programs in Teacher Education", en AILACTE Journal, año 3, Estados Unidos: The Association of Independent Liberal Arts Colleges for Teacher Education.

Fernsten, Linda (2009), "Teacher candidates, discourse and diversity", en Teacher Development, año 13, núm. 3, Reino Unido: Routledge.

Fox, Rebecca y Rosario Diaz-Greenberg (2006), "Culture, multiculturalism and foreign/ world language standards in U.S. teacher preparation programs: toward a discourse of dissonance", en European Journal of Teacher Education, año 29, núm. 3, Reino Unido: Routledge.

Gay, Geneva (2013), “Teaching to and through cultural diversity”, en Curriculum Inquiry, año 43, núm. 1, Reino Unido: Wiley.

Gayle-Evans, Guda y Deanna Michael (2006), “A study of pre-service teachers' awareness of multicultural issues”, en Multicultural Perspectives, año 8, núm. 1, Estados Unidos: Routledge.

Grant, Carl y Melissa Gibson (2011), "Diversity and teacher education. A historical perspective on research and policy", en Ball, Arnetha y Cynthia Tyson [comps.], Studying diversity in teacher education, Lanham: AERA.

Grant, Carl y Walter Secada (1990), "Preparing teachers for diversity", en Houston, Robert [comp.], Handbook of research on teacher education, New York: MacMillan.

Gorski, Paul (2006), "Complicity with conservatism: the de-politicizing of multicultural and intercultural education”, en Intercultural Education, año 17, núm. 2, Países Bajos: Routledge/IAIE.

Guidry, Jan y Patience Sowa (2005), "Preparing for multicultural schools: teacher candidates dialogue online with teachers from Egypt, Japan, Ghana and the U.S.", en Teacher Education Quartery, año 32, núm. 1, Estados Unidos: California Council on Teacher Education.

Hammerness, Karen et al. (2005), "How teachers learn and develop", en Darling-Hammond, Linda y John Bransford [comps.], Preparing teachers for a changing world: what teachers should learn and be able to do, San Francisco: Jossey-Bass.

He, Ye y Jewell Cooper (2009), "The ABCs for pre-service teacher cultural competency development”, en Teaching Education, año 20, núm. 3, Estados Unidos/Australia: Routledge. 
Henkin, Roxanne y Leann Steinmetz (2008), “The need for diversity education as perceived by preservice teachers", en Journal of the Scholarship of Teaching and Learning, año 8, núm. 1, Estados Unidos: The Trustees of Indiana University.

Herrada, Rosario Isabel (2010), "El concepto de raza según los futuros docentes. Experiencia formativa necesaria para enseñar en escuelas multiculturales”, en Enseñanza \& Teaching, año 28, núm. 2, España: Universidad de Salamanca.

Hill-Jackson, Valerie et al. (2007), "Having our say about multicultural education", en Kappa Delta Pi Record, año 43, núm. 4, Estados Unidos: Kappa Delta Pi.

Hollins, Etta y María Torres (2005), "Research on preparing teachers for diverse populations", en Cochran-Smith, Marilyn y Kenneth Zeichner [comps.], Studying teacher education. The Report of the AERA Panel on Research and Teacher Education, Washington: AERA.

Hsu, Hui-Yin (2009), "Preparing teachers to teach literacy in responsive ways that capitalize on students' cultural and linguistic backgrounds through Weblog technology", en Multicultural Education and Technology Journal, año 3, núm. 3, Reino Unido: Emerald.

Ibáñez, Nolfa et al. (2012), "La comprensión de la diversidad en interculturalidad y educación”, en Convergencia, Revista de Ciencias Sociales, núm. 59, México: Universidad Autónoma del Estado de México.

Kinloch, Valerie (2011), "Crossing boundaries, studying diversity: lessons from preservice teachers and urban youth", en Ball, Arnetha y Cynthia Tyson [comps.], Studying diversity in teacher education, Lanham: AERA.

Klug, Beverly et al. (2006), "Stepping out of our own skins: overcoming resistance of isolated preservice teacher populations to embracing diversity in educational settings", en Multicultural Perspectives, año 8, núm. 3, Estados Unidos: Routledge.

Kyles, Carli y Lori Olafson (2008), "Uncovering preservice teachers' beliefs about diversity through reflective writing”, en Urban Education, año 43, núm. 5, Estados Unidos: Sage.

Laughter, Judson (2011), "Rethinking assumptions of demographic privilege: diversity among white preservice teachers", en Teaching and Teacher Education, año 27, Estados Unidos: Elsevier.

Lenski, Susan Davis et al. (2005), "Beyond awareness: preparing culturally responsive preservice teachers”, en Teacher Education Quarterly, año 32, núm. 2, Estados Unidos: California Council on Teacher Education.

Liggett, Tonda (2011), "Critical multicultural education and teacher sense of agency”, en Teaching Education, año 22, núm. 2, Estados Unidos/Australia: Routledge.

Locke, Steven (2005), "Institutional social and cultural influences on the multicultural perspectives of preservice teachers", en Multicultural Perspectives, año 7, núm. 2, Estados Unidos: Routledge.

Lockhart, John (2010), “Critical investigations into interns' urban teaching apprenticeship experiences", en Dissertation Abstracts International, A: The Humanities and Social Sciences, año 70, núm. 10, Estados Unidos: University Microfilms International.

Marx, Helen y David Moss (2011), "Please mind the culture gap: intercultural development during a teacher education study abroad program", en Journal of Teacher Education, año 62, núm. 1, Estados Unidos: Sage.

Matus, Claudia y Marta Infante (2011), "Undoing diversity: knowledge and neoliberal discourses in colleges of education", en Discourse: studies in the cultural politics of education, año 32, núm. 3, Reino Unido/Australia: Routledge. 
McKnight, Anthony et al. (2011), "Using Slowmation for animated storytelling to represent non-Aboriginal preservice teachers' awareness of relatedness to country", en Australasian Journal of Educational Technology, año 27, núm. 1, Australia: Australasian Society for Computers in Learning in Tertiary Education.

Milner, Richard (2006), "Preservice teachers' learning about cultural and racial diversity: implications for urban education”, en Urban Education, año 41, núm. 4, Estados Unidos: Sage.

Moore, Felicia (2009), "Confronting assumptions, biases and stereotypes in preservice teachers' conceptualizations of science teaching through the use of book club”, en Journal of research in science teaching, año 46, núm. 9, Reino Unido: Wiley.

Morrier, Michael et al. (2007), "Teaching and learning within and across cultures: educator requirements across the United States”, en Multicultural Education, año 14, núm. 3, Estados Unidos: Caddo Gap Press.

Nero, Shondel (2009), "Inhabiting the other's world: language and cultural immersion for US-based teachers in the Dominican Republic", en Language, Culture and Curriculum, año 22, núm. 3, Reino Unido: Routledge.

OCDE (2010), Educating teachers for diversity. Meeting the challenge, París: OCDE/CERI. Pajares, Frank (1992), “Teachers' beliefs and educational research: cleaning up a messy construct", en Review of Educational Research, año 62, núm. 3, Estados Unidos: Sage.

Paredes, María Elisa (2009), Language attitudes, linguistic knowledge, and the multicultural education of pre-service teachers: A sociolinguistic study. Disertación doctoral, Estados Unidos: Universidad de Missouri, Saint Louis.

Patchen, Terri (2012), “'Little Rascals' in the 'City of God': film reflection and multicultural education”, en International Journal of Progressive Education, año 8, núm. 2, Estados Unidos: International Association of Educators.

Phillion, JoAnn et al. (2009), "Reimagining the curriculum: future teachers and study abroad", en Frontiers: The Interdisciplinary Journal of Study Abroad, año 18, Estados Unidos: The Forum on Education Abroad/Dickinson College.

Pinto, María (1989), "Introducción al análisis documental y sus niveles: el análisis de contenido", en Boletín de la $A N A B A D$, año 39, núm. 2, España: Federación Española de Asociaciones de Archiveros, Bibliotecarios, Arqueólogos, Museólogos y Documentalistas.

Reiter, Abigail y Shannon Davis (2011), "Factors influencing pre-service teachers' beliefs about student achievement: evaluation of a pre-service teacher diversity awareness program”, en Multicultural Education, año 19, núm. 3, Estados Unidos: Caddo Gap Press.

Richardson, Virginia y Peggy Placier (2001), “Teacher change”, en Richardson, Virginia [comp.], Handbook of Research on Teaching, Nueva York: American Educational Research Association.

Sales, Auxiliadora (2006), "La formación inicial del profesorado ante la diversidad: una propuesta metodológica para el nuevo Espacio Europeo de Educación Superior”, en Revista Interuniversitaria de Formación del Profesorado, año 20, núm. 3, España: AUFOP.

Settllage, John et al. (2009), "Constructing a doubt-free teaching self: self-efficacy, teacher identity and science instruction within diverse settings", en Journal of Research in science teaching, año 46, núm. 1, Reino Unido: Wiley.

Sleeter, Christine (2001), "Preparing teachers for culturally diverse schools. Research and the overwhelming presence of whiteness", en Journal of Teacher Education, año 52, núm. 2, Estados Unidos: Sage. 
Szabo, Susan y Gina Anderson (2009), "Helping teacher candidates examine their multicultural attitudes", en Educational Horizons, año 87, núm. 3, Estados Unidos: Sage.

Szecsi, Tunde et al. (2010), "Transforming teacher cultural landscapes by reflecting on multicultural literature”, en Multicultural Education, año 17, núm. 4, Estados Unidos: Caddo Gap Press.

Townsend, Tony y Richard Bates (2007), Handbook of Teacher Education, Países Bajos: Springer.

Trent, Stanley et al. (2008), "Preparing preservice educators for cultural diversity: how far have we come?", en Exceptional Children, año 74, núm. 3, Estados Unidos: The Council for Exceptional Children.

Tsigilis, Nikolaos et al. (2006), "Prospective early childhood educators' attitudes toward teaching multicultural classes: a planned behavior theory perspective", en Journal of Early Childhood Teacher Education, año 27, núm. 3, Estados Unidos: Taylor \& Francis.

Unesco (2009), Unesco World Report. Investing in Cultural Diversity and Intercultural Dialogue, París: Unesco.

Waddell, Jennifer (2011), "Crossing borders without leaving town: the impact of cultural immersion on the perceptions of teacher education candidates", en Issues in Teacher Education, año 20, núm. 2, Estados Unidos: Caddo Gap Press.

Wasonga, Teresa (2005), "Multicultural education knowledgebase, attitudes and preparedness for diversity", en International Journal of Educational Management, año 19, núm. 1, Reino Unido: Emerald.

Zeichner, Kenneth (2009), Teacher education and the struggle for social justice, New York: Routledge. Zippay, Cassie (2010), An exploration of the critical and reflective thinking and the culturally relevant literacy practices of two preservice teachers, Estados Unidos: Tennessee State University.

Zozakiewicz, Cathy (2010), "Culturally responsible mentoring: exploring the impact of an alternative approach for preparing student teachers for diversity", en The Teacher Educator, año 45, núm. 2, Estados Unidos: Ball State University/Taylor \& Francis.

Zygmunt-Fillwalk, Eva (2005), "Disequilibrium and reconstruction: the urban encounter as catalyst for preservice educators' cultural transformation", en Journal of Early Childhood Teacher Education, año 26, núm. 2, Estados Unidos: Taylor \& Francis.

Zygmunt-Fillwalk, Eva y Ann Leitze (2006), "Promising practices in preservice teacher preparation: The Ball State University urban semester", en Childhood Education, año 82, núm. 5, Estados Unidos: Association for Childhood Education International.

\section{Recursos electrónicos}

Gunn, Ann Marie Alberton (2011), Developing a culturally responsive literacy pedagogy: preservice teachers, teaching cases and postcard narratives. Disertación doctoral, Estados Unidos: Universidad de South Florida. Disponible en: http://pqdtopen.proquest. com/pqdtopen/doc/822778363.html?FMT=AI [28 de septiembre de 2013].

Rodríguez Izquierdo, Rosa María (2009), "La investigación sobre la educación intercultural en España”, en Archivos Analíticos de Políticas Educativas, año 17, núm. 4, Estados Unidos: University of South Florida/Mary Lou Fulton College of Education Arizona State University. Disponible en: http://www.redalyc.org/pdf/2750/275019727004. pdf [14 de octubre de 2013]. 


\section{Anexo}

\section{Cuadro 1}

\section{Revistas}

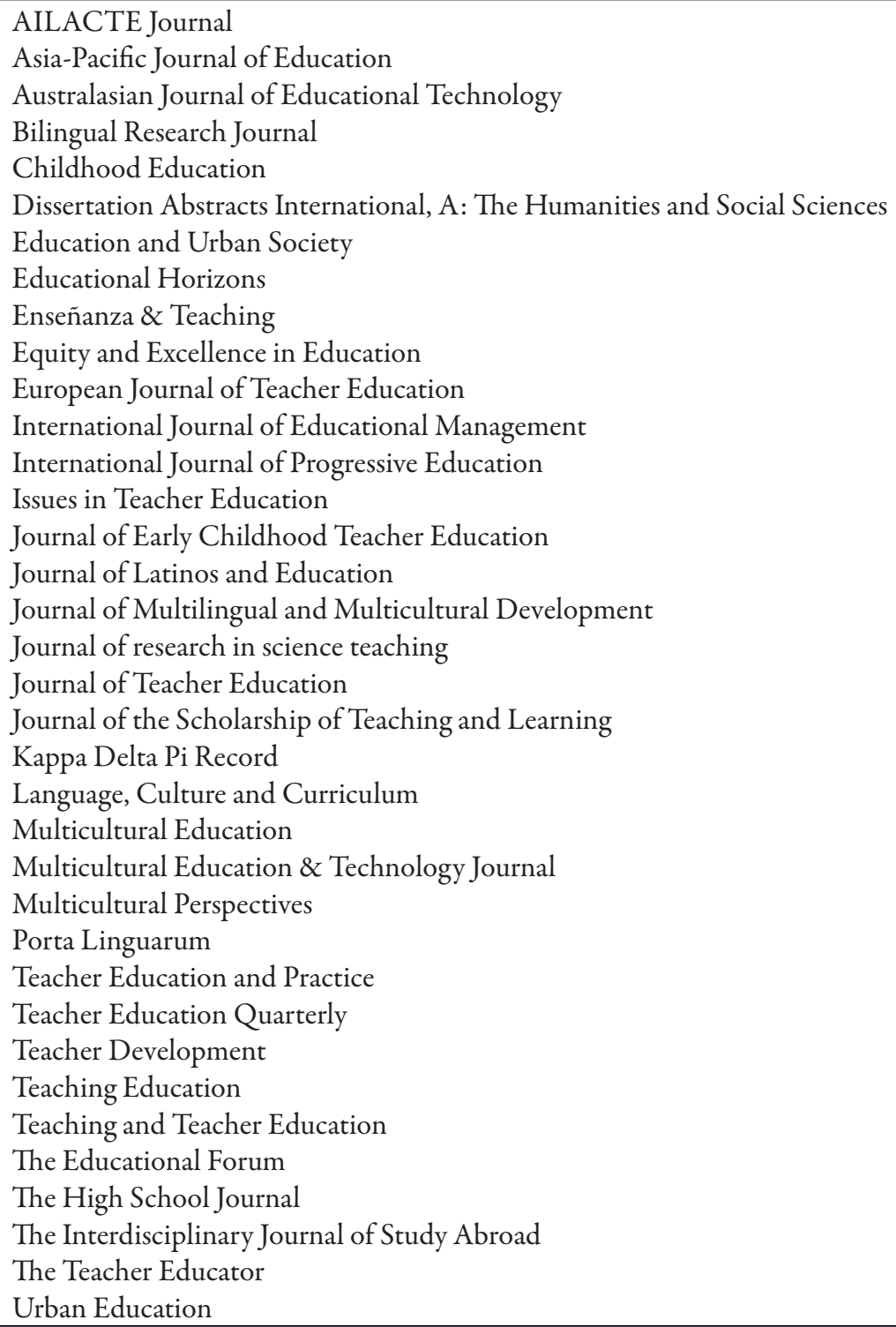


Eva Francisca Hinojosa Pareja. Doctora en Educación por la Universidad de Granada y profesora en el Departamento de Educación de la Universidad de Córdoba (España). Líneas de investigación: educación intercultural, convivencia y cultura de paz, formación inicial del profesorado, innovación docente. Publicaciones recientes: Muñoz, Juan Manuel, Eva Hinojosa y Esther Vega, "Opiniones de estudiantes universitarios acerca de la utilización de mapas mentales en dinámicas de aprendizaje cooperativo. Estudio comparativo entre la Universidad de Córdoba y La Sapienza de Roma”, en Perfiles Educativos (2015, pre-print); López, $\mathrm{M}^{\mathrm{a}}$ Carmen, Eva Hinojosa y Dolores Sánchez, "Evaluación de la calidad de los proyectos de innovación docente universitaria”, en Profesorado, Revista de currículum y formación del profesorado, año 18, núm. 3, España: FORCE/Universidad de Granada (2014); Arenas, María y Eva Hinojosa, "Análisis de impactos derivados de experiencias ecopedagógicas”, en Investigación en la Escuela, año 79, España: Diada Editora (2013).

$M^{a}$ Carmen López López. Doctora en Pedagogía por la Universidad de Granada y profesora titular en el Departamento de Didáctica y Organización Escolar de la Universidad de Granada (España). Líneas de investigación: formación inicial y desarrollo profesional del profesorado, educación intercultural, prácticum y didáctica universitaria. Publicaciones recientes: León, María José y Ma Carmen López, "Criterios para la Evaluación de los Proyectos de Innovación Docente Universitarios”, en Estudios sobre Educación, año 26, España: Universidad de Navarra (2014); Hinojosa, Eva, María Arenas y Ma Carmen López, "La Carta de la Tierra en Educación obligatoria desde una perspectiva Internacional", en Convergencia. Revista de Ciencias Sociales, año 21, núm. 66, México: UAEM (2014); Fernández Herrería, Alfonso y Ma Carmen López López, "Educar para la paz. Necesidad de un cambio epistemológico", en Convergencia. Revista de Ciencias Sociales, año 21, núm. 64, México: UAEM (2014).

Recepción: 16 de enero de 2015.

Aprobación: 15 de marzo de 2016. 
